\title{
The 6th Annual Toronto Breast Surgery Symposium, March 30, 2006, and the 36th Annual Symposium on Aesthetic Plastic Surgery, March 31-April 1, 2006
}

$\mathrm{T}$ The University of Toronto's Annual Breast Surgery Symposium, chaired by Dr M Brown and Dr J Semple, was followed by the Annual Symposium on Aesthetic Plastic Surgery, chaired by Dr W Peters.

The meeting was small enough to meet friends and share surgical experiences. Every meeting has pearls, and our invited guests were outstanding speakers and teachers. Rather than listing all the speakers, I will mention a few of the ideas discussed at the meeting.

Some breast reconstruction is being done with submuscular expanders and implants with very slow expansion. Nipple reconstruction is done alone and the areola is added later. If a breast cancer is in situ or low-grade, immediate breast reconstruction is more likely than if there are multiple positive axillary lymph nodes and radiation is used. The breast reconstruction rate is $10 \%$ and those who have reconstruction live longer. This fascinating finding could be related to immunity, well-being and surgeon selection.

Chest wall recurrence can have a good outcome. With wide excision, there are many flaps available for coverage: vertical rectus abdominis myocutaneous flaps, free flaps, large abdominal flaps and latissimus dorsi flaps. A dermis homograft can be used to add cover to an expander in immediate reconstructions sutured from the chest wall to the lower border of the pectoralis muscle. Breast implants can be safely irradiated and the skin below the inframammary crease can be dissected to recruit skin into the breast reconstruction. Immediate reconstructions are on the rise, partly because there is a considerable wait for delayed reconstructions, and because a transverse rectus abdominis myocutaneous flap surgery now requires only about three days in hospital after surgery, aided by printed patient information.

The breast implant debate goes on. Some surgeons prefer gel implants above muscle if one can pinch more than $2 \mathrm{~cm}$ in the upper pole for better implant cover, if there is slight ptosis and if the pectoralis is thick. Saline-filled implants, partially submuscular, are advised if there is less than a $2 \mathrm{~cm}$ pinch, if there is no ptosis and if the pectoralis is thin. The breast may look better with the transaxillary approach because the inferior pectoralis is intact.

Many of the tenets of breast augmentation were debated, such as 'bigger is better', achieving the patient's desired cup size, a specific implant making a specific breast cup size, further inflation for rippling, implants to treat ptosis, implants to produce cleavage, anatomic implants are superior and different implants can produce symmetry; all are commonly believed but can be challenged. Reconciling 'wishes with tissues' underlies the best decisions.

We discussed what 'cohesive' means. Essentially all gel-filled implants are cohesive; the difference is how cross-linked and how thick the gel is. We were reminded that breast implants can change the breast. Very big implants can cause breast tissue atrophy over time, and those who want very large implants should be advised against surgery; certainly the implications would need to be discussed in detail. Intensive preselection is the best predictor of patient happiness, and reoperation for size change should be discouraged. If a secondary size change operation is performed, an additional $50 \mathrm{~mL}$ to $100 \mathrm{~mL}$ will not be seen - a $200 \mathrm{~mL}$ difference will be needed. Accurate surgery and minimizing drugs can make augmentation recovery rapid.

In the face, the best view to assess facial youth is the threequarter view, which should show a gentle 'S' curve. This can be achieved by doing a superficial musculoaponeurotic system elevation above the jowl. A hollow upper eyelid looks old, so upper lid fat grafts below the eyebrow can rejuvenate. Fat grafting the nasojugal groove in the cheek is good, and a hollow temple can be augmented. Fat grafts can be used on each side of the centre of the lower lip to create areas of fullness, at the base of the nose and at the red-white margins of the lip and the chin. The lateral tarsal support suture is a useful addition to lower lid blepharoplasty. Fat grafts should not overcorrect, and fat at four months is permanent. Constant motion and multiple passes while injecting small amounts of fat are key details. Facial rejuvenation is not the application of one operation to all faces; rather, it is the application of multiple operations to achieve a subtle result and recovery in a reasonable time period.

In abdominoplasty, some surgeons advise against abdominal wall liposuction at the same time, but it is safe to perform as long as the lateral abdominal wall blood supply is intact. Lateral sutures can aid in narrowing the waist. Paralytic ileus can occur as a complication of abdominoplasty even without perforation.

Informed discharge is an accepted concept in clinical practice, particularly knowing what to look for postoperatively. Risk management is showing benefits in a decreasing number of claims.

It is this writer's view that the symposium owes its effectiveness to all those who participated and shared the practical aspects of breast and aesthetic surgery surgery.

John R Taylor

E-mail jrtaylor@ca.inter.net 